\title{
Atherogenic dyslipidemia and cardiovascular risk in children with nonalcoholic fatty liver disease
}

\author{
Naim Alkhouri ${ }^{1,2}$, Christine Carter-Kent ${ }^{1}$, Michael Elias ${ }^{1}$, and Ariel E Feldstein ${ }^{\dagger, 1}$ \\ ${ }^{1}$ Pediatric Gastroenterology, Lerner Research Institute Cleveland Clinic College of Medicine of \\ CWRU, OH, USA \\ 2Department of Pediatric Gastroenterology, Cleveland Clinic, 9500 Euclid Avenue, Cleveland, $\mathrm{OH}$ \\ 44195, USA
}

\begin{abstract}
Nonalcoholic fatty liver disease is now regarded as the most common form of chronic liver disease in adults and children. The close association between nonalcoholic fatty liver disease (NAFLD) and the metabolic syndrome has been extensively described. Moreover, a growing body of evidence suggest that NAFLD by itself confers a substantial cardiovascular risk independent of the other components of the metabolic syndrome. Given the significant potential for morbidity and mortality in these patients, and the large proportion of both pediatric and adult population affected, it is important that we clearly define the overall risk, identify early predictors for cardiovascular disease progression, and establish management strategies. In this article, we will focus on current data linking NAFLD and the severity of liver damage present in children with cardiovascular risk.
\end{abstract}

\section{Keywords}

cardiovascular disease; children; dyslipidemia; metabolic syndrome; nonalcoholic fatty liver disease

\section{Nonalcoholic fatty liver disease in children: epidemiology \& pathogenesis}

Nonalcoholic fatty liver disease (NAFLD) is a highly prevalent chronic liver disease in children in the USA and other parts of the world [1-3]. It is estimated that one in ten children in the USA have NAFLD [4]; however, the real prevalence of NAFLD in children remains largely unknown because of the lack of population-based studies and reliable noninvasive tests for screening.

The full histologic spectrum of NAFLD has been observed in children including simple steatosis with over-accumulation of lipids in the liver mostly in the form of triglycerides (TGs), to nonalcoholic steatohepatitis (NASH), which is characterized by fat accumulation along with hepatocyte injury, inflammation and a variable degree of fibrosis $[5,6]$. The main source of hepatic lipid loading is free fatty acids (FFAs) that are released into the circulation

\footnotetext{
(C) 2011 Future Medicine Ltd

${ }^{\dagger}$ Author for correspondence: Center for Cardiovascular Diagnostics \& Prevention, Cleveland Clinic, Cleveland, Ohio, USA, Tel.: +1 216444 5348, feldsta@ccf.org.

Financial \& competing interests disclosure: This work was supported by NIH grants (DK076852) and (DK082451) to Ariel E Feldstein. The authors have no other relevant affiliations or financial involvement with any organization or entity with a financial interest in or financial conflict with the subject matter or materials discussed in the manuscript apart from those disclosed.

No writing assistance was utilized in the production of this manuscript.
} 
from adipose tissue. Other sources include FFAs from dietary chylomicrons, de novo lipogenesis from glucose, decreased oxidation of FFAs in the mitochondria and alteration of TG export from the liver in the form of VLDL [7,8].

\section{NAFLD \& the metabolic syndrome: a new component or an independent risk factor?}

The rise in the prevalence of NAFLD corresponds to the epidemic of childhood obesity and the metabolic syndrome (MS). A landmark study by Sonia Caprio and her group demonstrated that the MS was very common among obese children and adolescents with prevalence reaching 50\% in severely obese children [9]. Plasma levels of C-reactive protein (CRP) and IL-6, which are potential predictors of adverse cardiovascular (CV) outcomes, correlated significantly with the degree of obesity, whereas adiponectin levels decreased with increasing obesity. More importantly, by using data from the Bogalusa Heart Study and the Cardiovascular Risk in Young Finns Study, Magnussen et al. showed that dichotomous definitions of MS in youth predicted important disease outcomes, such as adult MS, high carotid intima media thickness, and Type 2 diabetes mellitus (DM2) in early-to-middle adulthood [10].

Accumulating new evidence suggests that hepatic steatosis per se may confer independent metabolic consequences above and beyond the presence of obesity and MS. Data from the Framingham Heart Study revealed that patients with fatty liver had a higher risk of diabetes, hypertension, MS, higher TGs, and lower HDL and adiponectin levels independent of other fat depots (BMI, waist circumference and visceral adipose tissue) [11]. Moreover, Musso et $a l$. found that in nonobese nondiabetic subjects, NAFLD was more tightly associated with insulin resistance (IR) and markers of oxidative stress and endothelial dysfunction than the MS as defined by the Adult Treatment Panel (ATP) III. They suggested that NAFLD should be included in the definition of MS to help identify patients with increased cardiometabolic risk [12]. By using liver steatosis as an additional criterion to define pediatric MS, the prevalence of MS increased from 14 to $20 \%$ among white prepubertal obese children referred to an obesity clinic at the University of Chieti in Italy [13]. The same investigators compared two groups of obese adolescents with high hepatic fat fraction $(>5.5 \%)$ and low hepatic fat fraction measured by fast MRI and showed that fatty liver by itself was associated with impaired insulin sensitivity and early defects in $\beta$-cell function [14]. Several experimental studies using different animal models have also provided evidence that lipid accumulation in the liver leads to subacute hepatic inflammation and downstream cytokine production via the NF- $\mathrm{kB}$ pathway, which can cause IR both locally in the liver and systemically leading to the development of DM2 and MS [15]. An elegant animal study using liver insulin receptor knockout mice showed that purely hepatic IR was sufficient to induce a proatherogenic lipid profile and severe atherosclerosis [16].

\section{NAFLD, IR \& the adipocyte-hepatocyte crosstalk}

Adipose tissue in obese individuals is characterized by a state of low-grade chronic inflammation with accumulation of macrophages and the release of inflammatory cytokines including TNF- $\alpha$ and IL-6 [17-19]. Inflammation plays a central role in inducing IR through the activation of JNK, which inhibits insulin action by serine phosphorylation of insulin receptor substrate-1 [20,21]. IR induced by chronic inflammation is essential for the development of obesity-related complications including NAFLD, DM2, MS and CV disease. In fact, recent trials have evaluated the role of salicylate compounds for the treatment of DM2 through their anti-inflammatory effects [22-24]. High-dose salicylate inhibits the serum kinase IKK- $\beta$, which plays a significant role in tissue inflammation and IR through its fatty acid-dependent activation [25]. Hundal et al. studied nine patients with DM2 before 
and 2 weeks after treatment with high-dose aspirin (7g/day) and showed reduction in fasting plasma glucose, total cholesterol, TG and CRP, independent of body weight [24]. A randomized placebo-controlled trial has shown that salsalate, a pro-drug of salicylate, lowered hemoglobin A1C levels and improved other markers of glycemic control in adult patients with DM2 [26]. Furthermore, salsalate lowered TG levels and increased circulating adiponectin levels, which may predict decreased CV risk. These results should be interpreted with caution due to the relatively small sample size and the short follow-up period. The inhibition of macrophage infiltration into adipose tissue may protect against these complications. Recently, we have shown that adipocyte apoptosis may be an early event during the development of obesity that attracts macrophages to migrate into adipose tissue in both mice and humans [27]. Genetic inhibition of adipocyte apoptosis in a mouse model of obesity leads to a decrease in the number of macrophages in adipose tissue and the protection of these animals from IR, dyslipidemia and hepatic steatosis. Apoptosis can be manipulated by new therapeutic agents including caspase inhibitors and this may represent a new strategy for treating obesity-related complications that predispose to $\mathrm{CV}$ disease.

\section{Markers of atherosclerosis \& CV complications in NAFLD}

The strong association between NAFLD, IR and MS has stimulated an interest in the potential role of NAFLD in the pathogenesis of CV disease. There is a growing body of evidence suggesting an association between NAFLD and CV disease that goes beyond what would be expected by the presence of MS alone [28]. Moreover, patients with the advanced form of NAFLD, NASH, may be at higher risk for atherosclerosis than patients with simple steatosis. This is thought to be due to multiple possible mechanisms including increased oxidative stress and systemic inflammation. CV risk can be assessed by different modalities including the presence of an atherogenic lipid profile, levels of inflammatory markers, markers of endothelial dysfunction and epidemiologic studies evaluating the actual prevalence or incidence of CV disease in patients with NAFLD (Figure 1).

\section{NAFLD \& atherogenic dyslipidemia}

Atherogenic dyslipidemia characterized by high TGs and low HDL-C concentrations is common in obese children and adolescents. As a matter of fact, the Third National Health and Nutritional Examination Survey (NHANES) III showed that the most common components of the MS in adolescents were high TG and low HDL levels [29]. Plasma lipid levels varied by race and ethnicity, with hypertriglyceridemia and low HDL levels being most common among non-Hispanic white people and least common among non-Hispanic black people. Moreover, increased levels of small dense LDL, another important risk factor for future coronary heart disease mortality and morbidity, appears to be commonly present in obese children [30,31]. The fundamental defect that is thought to lead to this atherogenic lipid profile is the overproduction of larger TG-rich VLDL particles by the liver [32]. Normally, insulin inhibits adipose tissue lipolysis and hepatic VLDL secretion. In obesityinduced IR, there is an increased flux of FFA from adipose tissue to the liver, which can be re-esterified into TG, and increased VLDL secretion by the liver [33]. Other mechanisms of hepatic lipid loading include de novo lipogenesis from glucose and decreased FFA oxidation at the level of the mitochondria [34]. Recent studies have suggested a genetic basis for the abnormal lipoprotein metabolism in patients with NAFLD. Genome-wide association studies demonstrated that a genetic variation in adiponutrin/patatin-like phospholipase-3 (PNPLA3) confers susceptibility to NAFLD [35]. Adiponutrin is expressed in the liver and adipose tissue and has lipase activity against TG. The loss of function variant (PNPLA3 rs738409) predisposes a patient to steatosis by decreasing TG hydrolysis in liver cells [36]. Obese patients often have low HDL-C levels even when fasting TG levels are normal, suggesting that other mechanisms in obesity contribute to the low HDL-C levels beyond simply elevation in TG-rich lipoproteins such as increased production of cholesteryl ester 
transfer protein by adipose tissue and increased activity of hepatic lipase, as reviewed elsewhere [33].

Ratios of cholesterol ester-rich lipoproteins such as total cholesterol/HDL and LDL/HDL are well established risk factors for CV disease. More recently TG-rich lipoproteins (chylomicrons, chylomicron remnants and VLDL) have been shown to play a role in atherogenesis $[37,38]$. The TG/HDL ratio correlates inversely with the plasma level of small, dense LDL particles. This ratio is a powerful independent predictor of coronary artery disease development, even stronger than TC/HDL and LDL/HDL ratios $[39,40]$.

Apolipoprotein (Apo)B is the primary protein component of the atherogenic LDL particles, whereas ApoA1 is the primary protein component of the anti-atherogenic HDL particles. The ratio of ApoB to ApoA1 reflects the balance of cholesterol transport and the higher the value of this ratio, the more cholesterol is likely to be deposited in the arterial wall $[41,42]$. ApoB, ApoA1 and the ApoB:ApoA1 ratio have been reported as better predictors of CV events than LDL-C and they even retain their predictive power in patients receiving lipidmodifying therapy. Measurement of these apolipoproteins could improve CV risk prediction. Moreover, a recent study demonstrated that the ApoB:ApoA1 ratio was strongly associated with the presence of individual MS components, with the MS itself and with IR [43]. However, both ApoB and ApoA1 are produced in the liver and their production might be altered in patients with chronic liver disease including NAFLD [44].

A study of 67 prepubertal children showed that TG/HDL ratio was increased in obese children and it correlated with elevated mean blood pressure and the presence of MS [45]. A TG:HDL ratio $\geq 3$ was associated with lower insulin sensitivity and higher visceral fat in another study of 35 overweight adolescents, with a sensitivity of $61 \%$ and a specificity of $82 \%$ for identifying participants with the greatest degree of IR [46]. This ratio could be a useful tool in identifying children at risk for dyslipidemia, hypertension and the MS [45]. In a case-control study of 150 children with NAFLD and 150 overweight children without NAFLD, Schwimmer et al. found that children with biopsy-proven NAFLD had significantly higher total cholesterol, LDL and TG, and lower HDL levels [47]. In a recent study, Cali et al. found that hepato-steatosis in adolescents, identified by fast MRI, was associated with a markedly "proatherogenic lipoprotein profile" characterized by small dense LDL, larger VLDL and decreased large HDL concentrations [48]. Although there was no difference in the total number of LDL particles between adolescents with and without hepatosteatosis, those with hepatic steatosis had significantly higher concentrations of small dense LDL. Likewise, total HDL particle concentration was not different between the two groups, but adolescents with hepatosteatosis had significantly higher concentrations of large HDL and lower concentrations of medium HDL particles compared with those without hepatosteatosis. Overall size of the lipoproteins followed a similar trend. This suggests that the ApoB:ApoA1 ratio, which reflects the total number of LDL and HDL particles, may not be a useful marker for CV risk in patients with NAFLD.

We took the previous findings a step further by demonstrating that the histological severity of NAFLD as assessed by NAFLD Activity Score and fibrosis score correlated with higher traditional lipid ratios and a more atherogenic lipid panel in a recent study of 118 children with biopsy-proven NAFLD [49]. This suggests that patients with more advanced disease (NASH and fibrosis) may be at a higher risk for atherosclerosis and CV disease compared with patients with simple steatosis. The ApoB:ApoA1 ratio did not show the same correlation with liver histology as the other lipid ratios. We have shown similar results in another study of a group of adult patients with the full histologic spectrum of NAFLD. NASH was associated with higher lipid ratios independent of obesity, IR and the presence of diabetes [50]. 
The relative size of HDL and its composition determine its anti-atherogenic properties with large $\mathrm{HDL}_{2}$ being protective against atherosclerosis and small $\mathrm{HDL}_{3}$ representing a $\mathrm{CV}$ risk factor. Patients with fatty liver have lower circulating levels of $\mathrm{HDL}_{2}$ and $\mathrm{HDL}_{2}: \mathrm{HDL}_{3}$ ratio compared with controls suggesting an increased risk for $\mathrm{CV}$ disease [51]. The association between liver fat and $\mathrm{HDL}_{2}$ and $\mathrm{HDL}_{2}: \mathrm{HDL}_{3}$ ratio remained statistically significant after adjusting for IR and adiponectin levels. HDL size distribution is shifted towards small HDL particles in children with IR, DM2 and the MS [52,53]. Adolescents with hepatic steatosis were found to have decreased levels of the large protective HDL [48]. These data further imply that determination of $\mathrm{HDL}_{2}-\mathrm{C}$, in addition to total $\mathrm{HDL}-\mathrm{C}$, may more precisely predict the actual risk for $\mathrm{CV}$ events in subjects with fatty liver. Collectively, the previous studies support the presence of an important link between hepatic steatosis/liver injury and the development of an atherogenic lipid profile in children with NAFLD.

\section{NAFLD \& systemic inflammation}

Nonalcoholic fatty liver disease is associated with a state of chronic inflammation and increased oxidative stress, which may play a role in the pathogenesis of atherosclerosis. Patients with NAFLD have higher levels of high-sensitivity CRP, TNF- $\alpha$, oxidized LDL and plasma plasminogen activator inhibitor-1 and lower levels of adiponectin than controls [5458]. Moreover, inflammatory cytokines tend to be higher in patients with NASH compared with patients with simple steatosis. Plasma levels of CRP, which is an indicator of systemic inflammation and a risk factor for atherosclerosis [59], were found to be higher in patients with histologically proven NASH [60]. A large study that included 400 children showed that there was a stepwise increase in high-sensitivity CRP levels from healthy controls to obese children with normal liver to obese children with NAFLD [61]. Data from Nobili and colleagues showed that circulating levels of the inflammatory cytokine TNF- $\alpha$ correlated with histologic liver injury scores in children with NAFLD [62]. Adiponectin is another cytokine secreted from adipose tissue with anti-atherogenic effects [63,64]. Adiponectin levels have been shown to correlate negatively with early markers of atherosclerosis and the MS in obese children [65]. More importantly, adiponectin levels are lower in children with NAFLD compared with lean controls, which may increase their risk for developing atherosclerosis [66]. Bugianesi et al. showed that decreased levels of circulating adiponectin in NAFLD is related to hepatic insulin sensitivity and to the amount of hepatic fat content but not to fibrosis or necroinflammation [67].

More recently the neutrophil:lymphocyte (N/L) ratio has emerged as a prognostic marker in patients with CV disease [68]. This ratio integrates information on two different immune pathways, the neutrophils, which are responsible for ongoing inflammation, and the lymphocytes, which represent the regulatory pathway. Thus, a higher N/L ratio is an indicator of the overall inflammatory status of the body. This ratio has been validated as a predictor of outcome in multiple studies in patients with coronary artery disease [68-71]. Nascimento et al. demonstrated a significant change in the differential leukocyte count toward neutrophilia in obese patients aged 6-12 years old [72]. We have conducted a study to examine the relationship between N/L ratio and histologic severity of NAFLD in adults and found that this ratio was higher in patients with NASH and advanced fibrosis compared with patients with simple steatosis indicating that the patients with advanced disease may have a higher CV risk [73]. Another validated marker of atherosclerosis that can be easily obtained from routine blood counts is the mean platelet volume (MPV), which reflects platelet activation and aggregation [74,75]. In a study of 128 obese adolescents, MPV was significantly higher in obese patients with NAFLD compared with obese patients without NAFLD or controls. This indicates that children with NAFLD have higher CV risk; however, correlation with liver histology was not examined as the diagnosis of NAFLD was made based on ultrasound findings and the presence of elevated liver enzymes [76]. 


\section{NAFLD \& early vascular changes}

Multiple cross-sectional studies have shown a significant increase in carotid intima-media thickness (IMT), as a marker of early atherosclerosis, in patients with NAFLD [77,78]. A recent meta-analysis of seven studies that included 3497 patients showed that NAFLD is strongly associated with carotid IMT and the presence of carotid plaques [79]. Furthermore, the histological severity of NAFLD has been shown to independently predict carotid IMT with significantly higher values in patients with NASH compared with patients with simple steatosis [80]. In a study by Villanova et al., patients with NAFLD had endothelial dysfunction as assessed by brachial artery flow-mediated dilation and the severity of liver disease was associated with more altered endothelial function [81].

Several studies in children have attempted to evaluate the relationship between early atherosclerosis and NAFLD. Pacifico et al. found that the severity of fatty liver as assessed by ultrasound was significantly associated with maximum carotid IMT after adjusting for age, gender, Tanner stage and classic CV risk factors [82]. Another study in a pediatric population found carotid IMT to be significantly higher in obese children with hepatosteatosis compared with healthy children or obese children without hepatosteatosis [83]. Kelishadi et al. found that carotid IMT was significantly associated with IR and NAFLD in adolescents suggesting that the liver and the vessels share common mediators [84]. The previous findings were confirmed in a large pediatric study assessing the relationship between NAFLD during childhood and CV risk factors [61]. Children with NAFLD ( $\mathrm{n}=$ 100) had decreased flow-mediated dilation of the brachial artery and increased carotid IMT compared with obese children without fatty liver $(n=150)$ and healthy controls $(n=150)$.

Furthermore, autopsy findings in 817 children who died of external causes showed that atherosclerosis was significantly more common in children with fatty liver than those without it (30 vs 19\%, respectively) [85]. Multislice computed tomography is another noninvasive method to directly detect and classify plaques in the coronary arteries [86] and to determine which ones are vulnerable to rupture, which can cause acute coronary syndrome. Patients with NAFLD were found to be at higher risk of having vulnerable plaque as detected by multislice computed tomography independent of classic risk factors for $\mathrm{CV}$ disease or individual components of the MS [87].

Adhesion molecules such as ICAM-1 and VCAM play an important role in leukocyte interaction with the endothelium and migration into the artery wall as a part of the atherogenic process. Circulating levels of these molecules may reflect endothelial dysfunction and indicate increased risk for CV disease and they have been described to be elevated in patients with the MS [88]. Studies assessing the levels of these adhesion molecules in NAFLD patients have given controversial results. One study in particular showed that NAFLD patients have higher circulating levels of biomarkers of endothelial dysfunction, namely soluble (s)ICAM-1, sCD40 ligand and plasminogen activator inhibitor-1 [89]. In this study, hepatic expression of these molecules by immunostaining correlated with the degree of liver steatosis and necroinflammation. In contrast, a recent study by Lucero et al. showed no difference in SICAM or SVCAM levels when comparing patients with the MS with and without fatty liver [90]. Ito et al. demonstrated no difference in sICAM-1 between patients with NAFLD and controls; however, there was a marked increase in patients with NASH [91] indicating that the degree of liver injury and inflammation may be a more important factor in the pathogenesis of atherosclerosis than just the mere presence of fatty liver.

Taken together, these studies suggest that NAFLD and the degree of liver damage and inflammation present in both adults and pediatric patients are strongly associated with endothelial dysfunction and early atherosclerosis. 


\section{Epidemiologic studies}

Numerous epidemiologic studies have clearly demonstrated an increase in the prevalence of CV disease in patients with NAFLD. The mortality rate in patients with NAFLD is higher than the general population, most commonly due to CV disease and malignancy $[92,93]$. Sanyal et al. demonstrated that heart disease contributed disproportionately to mortality in patients with cirrhosis due to NASH compared with those with hepatitis C infection [94]. Diabetic patients with NAFLD diagnosed by ultrasound had an increased incidence of CV events compared with diabetic patients without NAFLD [78]. The notion that hepatic inflammation by itself is atherogenic is supported by the observation that $\mathrm{CV}$ disease is strongly associated with elevated alanine aminotransferase and $\gamma$-glutamyltransferease, which are surrogate markers of liver necroinflammation [95-97]. Two large pediatric studies showed an association between elevated serum alanine transaminase levels and the prevalence of the MS and IR as markers of CV risk in obese children aged 3-18 years $[98,99]$. Longitudinal studies are needed to assess the development of adulthood CV disease in these children with abnormal liver enzymes and other risk factors for atherosclerosis.

\section{Future perspective}

As we learn more about the associations between NAFLD, dyslipidemia and CV disease, it has become clear that the potential morbidity to adults and children alike is significant. Moving forward, it will be important to determine if abnormal lipid profiles and increased CV inflammatory mediators in children with NAFLD translate into early CV events including myocardial infarction and stroke. The aforementioned studies regarding the detection of increased carotid IMT in patients with NAFLD give some clues as to what lies ahead for children and adolescences diagnosed with NAFLD.

In addition, it will be important to determine if medical treatments that improve hepatic steatosis and reduce hepatic inflammation also impact the course of $\mathrm{CV}$ disease in these patients. It has already been shown that lifestyle interventions, if sustained, can have a significant impact on NAFLD. In their 2009 study of 152 adults, St. George et al. documented improvement in liver enzymes, insulin levels, total cholesterol and LDL after 3 months of moderate-intensity lifestyle counseling [100]. In one of the only randomized, controlled trials examining the effects of weight loss on NAFLD after intensive lifestyle modification, it was reported that after 48 weeks of intervention, patients who received intensive lifestyle modification lost $9.3 \%$ of their body weight as compared with $0.2 \%$ loss in control subjects. Importantly, in addition to weight loss, patients experienced improvement in the NAFLD Activity Score as well as reduction in circulating liver enzymes [100]. In children, Nobili et al. showed that after 12 months of lifestyle intervention, which included both a tailored diet and increased physical activity, improvement in NAFLD histology, lipid profiles and insulin levels were noted [101]. In the future, it may also be helpful to track serologic indicators of CV risk in response to lifestyle interventions for this cohort of children as well. Thus, we will be able to determine if these types of therapies modify $\mathrm{CV}$ risk over time.

Finally, medical therapies targeted at the reduction of mediators of inflammation and/or apoptosis could potentially lead to improvement in the liver damage seen in NASH, but could also lead to the reduction of the CV changes seen in these patients. Potential therapeutic agents of interest might include medications aimed at the reduction of apoptosis, such as caspase inhibitors, the reduction of pro-inflammatory cytokines such as anti-TNF medications or medications that decrease levels of $\mathrm{AboB}$ or the ApoB:ApoA1 ratio, thereby leading to a reduction of the formation of atherogenic LDL particles. Thus far, proven effective and safe pharmacological therapies for NAFLD are lacking; however, there is currently ongoing intense research in these areas. The results of the Treatment of 
Nonalcoholic Fatty Liver Disease in Children (TONIC) trial, which compares the use of vitamin $\mathrm{E}$ or metformin with placebo in the treatment of pediatric NAFLD, have been recently presented at the American Association for the Study of Liver Disease Annual meeting [102]. Neither vitamin E nor metformin demonstrated superiority over placebo for reaching the primary outcome of sustained reduction in alanine aminotransferase. However, vitamin E significantly improved important histological features including hepatocyte ballooning. Other potential therapeutic approaches include omega-3 fatty acids, antioxidants, anti-inflammatory agents such as TNF- $\alpha$ inhibitors, and statins.

\section{Conclusion}

It has become clear that NAFLD and the advanced form, NASH, are closely associated with the MS, IR and dyslipidemia in overweight and obese children and adults. This association is due to multiple factors including IR induced by inflammatory pathways and the downstream effects of IR on various organ systems. One of the most important considerations for children with NAFLD and co-existent MS are the potential complications that may develop and become life-threatening including liver fibrosis, end-stage liver disease, hypertensive crises and renal failure. A screening for fatty liver disease, either noninvasively or in selected cases with liver biopsies, should be incorporated into our routine evaluation in children and adolescents who are obese or have the MS. In addition, the risk of $\mathrm{CV}$ disease is concerning in these patients as well. Here we have discussed that patients with the MS are at a high risk for CV disease, but patients with NASH may be at an increased risk beyond that which would be expected for patients with the MS alone. Evidence for these findings includes multiple studies documenting the presence of atherogenic dyslipidemia in patients with NAFLD, higher circulating levels of pro-inflammatory cytokines and markers of oxidative stress, as well as evidence that newer prognostic markers of CV risk, such as the N/L ratio and MPV, are higher in patients with NAFLD. We have also detailed epidemio-logical studies showing higher rates of mortality from CV complications in patients with NAFLD.

Taking these findings into consideration, it is important that we continue to identify and refine the tests available to identify patients with NAFLD who are at risk for the development of $\mathrm{CV}$ disease so that they may be monitored closely and the proper intervention can take place when problems arise. Also, we continue to stress prevention in obese children before components of the MS or NAFLD arise. Pediatricians and pediatric specialists need to be vigilant in identifying patients who are obese or at risk for becoming obese and initiating early screening and intervention.

\section{Bibliography}

Papers of special note have been highlighted as:

- of interest

- of considerable interest

1. Chan DF, Li AM, Chu WC, et al. Hepatic steatosis in obese Chinese children. Int J Obes Relat Metab Disord. 2004; 28:1257-1263. [PubMed: 15278103]

2. Wieckowska A, Feldstein AE. Nonalcoholic fatty liver disease in the pediatric population: a review. Curr Opin Pediatr. 2005; 17:636-641. [PubMed: 16160540]

3. Wiegand S, Keller KM, Robl M, et al. Obese boys at increased risk for nonalcoholic liver disease: evaluation of 16,390 overweight or obese children and adolescents. Int J Obes (Lond). 2010; 34:1468-1474. [PubMed: 20531349] 
4. Schwimmer JB, Deutsch R, Kahen T, Lavine JE, Stanley C, Behling C. Prevalence of fatty liver in children and adolescents. Pediatrics. 2006; 118:1388-1393. [PubMed: 17015527]

5. Brunt EM. Pathology of fatty liver disease. Mod Pathol. 2007; 20(Suppl. 1):S40-S48. [PubMed: 17486051]

6. Feldstein AE, Charatcharoenwitthaya P, Treeprasertsuk S, Benson JT, Enders FB, Angulo P. The natural history of non-alcoholic fatty liver disease in children: a follow-up study for up to 20 years. Gut. 2009; 58:1538-1544. [PubMed: 19625277]

7. Browning JD, Horton JD. Molecular mediators of hepatic steatosis and liver injury. J Clin Invest. 2004; 114:147-152. [PubMed: 15254578]

8. Donnelly KL, Smith CI, Schwarzenberg SJ, Jessurun J, Boldt MD, Parks EJ. Sources of fatty acids stored in liver and secreted via lipoproteins in patients with nonalcoholic fatty liver disease. J Clin Invest. 2005; 115:1343-1351. [PubMed: 15864352]

9. Weiss R, Dziura J, Burgert TS, et al. Obesity and the metabolic syndrome in children and adolescents. N Engl J Med. 2004; 350:2362-2374. Important study showing that the metabolic syndrome is very common among obese children and adolescents with prevalence reaching $50 \%$ in severely obese children. [PubMed: 15175438]

10. Magnussen CG, Koskinen J, Chen W, et al. Pediatric metabolic syndrome predicts adulthood metabolic syndrome, subclinical atherosclerosis, and Type 2 diabetes mellitus but is no better than body mass index alone: the Bogalusa Heart Study and the Cardiovascular Risk in Young Finns Study. Circulation. 2010; 122:1604-1611. [PubMed: 20921439]

11. Speliotes EK, Massaro JM, Hoffmann U, et al. Fatty liver is associated with dyslipidemia and dysglycemia independent of visceral fat: the Framingham Heart Study. Hepatology. 2010; 51:1979-1987. [PubMed: 20336705]

12. Musso G, Gambino R, Bo S, et al. Should nonalcoholic fatty liver disease be included in the definition of metabolic syndrome? A cross-sectional comparison with Adult Treatment Panel III criteria in nonobese nondiabetic subjects. Diabetes Care. 2008; 31:562-568. [PubMed: 18056890]

13. D'Adamo E, Marcovecchio ML, Giannini C, et al. The possible role of liver steatosis in defining metabolic syndrome in prepubertal children. Metabolism. 2010; 59:671-676. [PubMed: 19913850]

14. D'Adamo E, Cali AM, Weiss R, et al. Central role of fatty liver in the pathogenesis of insulin resistance in obese adolescents. Diabetes Care. 2010; 33:1817-1822. [PubMed: 20668154]

15. Cai D, Yuan M, Frantz DF, et al. Local and systemic insulin resistance resulting from hepatic activation of IKK- $\beta$ and NF- $\kappa$ B. Nat Med. 2005; 11:183-190. [PubMed: 15685173]

16. Biddinger SB, Hernandez-Ono A, Rask-Madsen C, et al. Hepatic insulin resistance is sufficient to produce dyslipidemia and susceptibility to atherosclerosis. Cell Metab. 2008; 7:125-134. [PubMed: 18249172]

17. Weisberg SP, McCann D, Desai M, Rosenbaum M, Leibel RL, Ferrante AW Jr. Obesity is associated with macrophage accumulation in adipose tissue. J Clin Invest. 2003; 112:1796-1808. [PubMed: 14679176]

18. Hotamisligil GS. Inflammation and metabolic disorders. Nature. 2006; 444:860-867. [PubMed: 17167474]

19. Neels JG, Olefsky JM. Inflamed fat: what starts the fire? J Clin Invest. 2006; 116:33-35. [PubMed: 16395402]

20. Hirosumi J, Tuncman G, Chang L, et al. A central role for JNK in obesity and insulin resistance. Nature. 2002; 420:333-336. [PubMed: 12447443]

21. Tuncman G, Hirosumi J, Solinas G, Chang L, Karin M, Hotamisligil GS. Functional in vivo interactions between JNK1 and JNK2 isoforms in obesity and insulin resistance. Proc Natl Acad Sci USA. 2006; 103:10741-10746. [PubMed: 16818881]

22. Fleischman A, Shoelson SE, Bernier R, Goldfine AB. Salsalate improves glycemia and inflammatory parameters in obese young adults. Diabetes Care. 2008; 31:289-294. [PubMed: 17959861]

23. Goldfine AB, Silver R, Aldhahi W, et al. Use of salsalate to target inflammation in the treatment of insulin resistance and Type 2 diabetes. Clin Transl Sci. 2008; 1:36-43. [PubMed: 19337387]

24. Hundal RS, Petersen KF, Mayerson AB, et al. Mechanism by which high-dose aspirin improves glucose metabolism in Type 2 diabetes. J Clin Invest. 2002; 109:1321-1326. [PubMed: 12021247] 
25. Yuan M, Konstantopoulos N, Lee J, et al. Reversal of obesity- and diet-induced insulin resistance with salicylates or targeted disruption of Ikk $\beta$. Science. 2001; 293:1673-1677. [PubMed: 11533494]

26. Goldfine AB, Fonseca V, Jablonski KA, Pyle L, Staten MA, Shoelson SE. The effects of salsalate on glycemic control in patients with Type 2 diabetes: a randomized trial. Ann Intern Med. 2010; 152:346-357. [PubMed: 20231565]

27-. Alkhouri N, Gornicka A, Berk MP, et al. Adipocyte apoptosis: a link between obesity, insulin resistance and hepatic steatosis. J Biol Chem. 2010; 285:3428-3438. First study to implicate adipocyte apoptosis as an early event during the development of obesity-related inflammation. [PubMed: 19940134]

28. Targher G. Non-alcoholic fatty liver disease, the metabolic syndrome and the risk of cardiovascular disease: the plot thickens. Diabet Med. 2007; 24:1-6. [PubMed: 17227317]

29. de Ferranti SD, Gauvreau K, Ludwig DS, Neufeld EJ, Newburger JW, Rifai N. Prevalence of the metabolic syndrome in American adolescents: findings from the Third National Health and Nutrition Examination Survey. Circulation. 2004; 110:2494-2497. [PubMed: 15477412]

30. Kang HS, Gutin B, Barbeau P, Litaker MS, Allison J, Le NA. Low-density lipoprotein particle size, central obesity, cardiovascular fitness, and insulin resistance syndrome markers in obese youths. Int J Obes Relat Metab Disord. 2002; 26:1030-1035. [PubMed: 12119567]

31. Miyashita M, Okada T, Kuromori Y, Harada K. LDL particle size, fat distribution and insulin resistance in obese children. Eur J Clin Nutr. 2006; 60:416-420. [PubMed: 16278685]

32. Adiels M, Olofsson SO, Taskinen MR, Boren J. Overproduction of very low-density lipoproteins is the hallmark of the dyslipidemia in the metabolic syndrome. Arterioscler Thromb Vasc Biol. 2008; 28:1225-1236. [PubMed: 18565848]

33. Bamba V, Rader DJ. Obesity and atherogenic dyslipidemia. Gastroenterology. 2007; 132:21812190. [PubMed: 17498511]

34. Alkhouri N, Dixon LJ, Feldstein AE. Lipotoxicity in nonalcoholic fatty liver disease: not all lipids are created equal. Expert Rev Gastroenterol Hepatol. 2009; 3:445-451. [PubMed: 19673631]

35. Romeo S, Kozlitina J, Xing C, et al. Genetic variation in PNPLA3 confers susceptibility to nonalcoholic fatty liver disease. Nat Genet. 2008; 40:1461-1465. [PubMed: 18820647]

36. Valenti L, Al-Serri A, Daly AK, et al. Homozygosity for the patatin-like phospholipase-3/ adiponutrin I148M polymorphism influences liver fibrosis in patients with nonalcoholic fatty liver disease. Hepatology. 2010; 51:1209-1217. [PubMed: 20373368]

37. Gaziano JM, Hennekens CH, O'Donnell CJ, Breslow JL, Buring JE. Fasting triglycerides, highdensity lipoprotein, and risk of myocardial infarction. Circulation. 1997; 96:2520-2525. [PubMed: 9355888]

38. Hodis HN, Mack WJ. Triglyceride-rich lipoproteins and the progression of coronary artery disease. Curr Opin Lipidol. 1995; 6:209-214. [PubMed: 7670749]

39. da Luz PL, Cesena FH, Favarato D, Cerqueira ES. Comparison of serum lipid values in patients with coronary artery disease at $<50,50$ to 59,60 to 69 , and $>70$ years of age. Am J Cardiol. 2005; 96:1640-1643. [PubMed: 16360350]

40. da Luz PL, Favarato D, Faria-Neto JR Jr, Lemos P, Chagas AC. High ratio of triglycerides to HDL-cholesterol predicts extensive coronary disease. Clinics. 2008; 63:427-432. [PubMed: 18719750]

41. Walldius G, Jungner I. Apolipoprotein B and apolipoprotein A-I: risk indicators of coronary heart disease and targets for lipid-modifying therapy. J Intern Med. 2004; 255:188-205. [PubMed: 14746556]

42. Holme I, Aastveit AH, Hammar N, Jungner I, Walldius G. Relationships between lipoprotein components and risk of ischaemic and haemorrhagic stroke in the Apolipoprotein MOrtality RISk study (AMORIS). J Intern Med. 2009; 265(2):275-287. [PubMed: 19019184]

43. Sierra-Johnson J, Somers VK, Kuniyoshi FH, et al. Comparison of apolipoprotein-B/ apolipoprotein-AI in subjects with versus without the metabolic syndrome. Am J Cardiol. 2006; 98:1369-1373. [PubMed: 17134631] 
44. Fabbrini E, Mohammed BS, Magkos F, Korenblat KM, Patterson BW, Klein S. Alterations in adipose tissue and hepatic lipid kinetics in obese men and women with nonalcoholic fatty liver disease. Gastroenterology. 2008; 134:424-431. [PubMed: 18242210]

45. Quijada Z, Paoli M, Zerpa Y, et al. The triglyceride/HDL-cholesterol ratio as a marker of cardiovascular risk in obese children; association with traditional and emergent risk factors. Pediatr Diabetes. 2008; 9:464-471. [PubMed: 18507788]

46. Hannon TS, Bacha F, Lee SJ, Janosky J, Arslanian SA. Use of markers of dyslipidemia to identify overweight youth with insulin resistance. Pediatr Diabetes. 2006; 7:260-266. [PubMed: 17054447]

47. Schwimmer JB, Pardee PE, Lavine JE, Blumkin AK, Cook S. Cardiovascular risk factors and the metabolic syndrome in pediatric nonalcoholic fatty liver disease. Circulation. 2008; 118:277-283. [PubMed: 18591439]

48--. Cali AM, Zern TL, Taksali SE, et al. Intrahepatic fat accumulation and alterations in lipoprotein composition in obese adolescents: a perfect proatherogenic state. Diabetes Care. 2007; 30:30933098. Demonstrates that children with fatty liver have a proatherogenic lipoprotein profile characterized by small dense LDL, larger VLDL and decreased large HDL concentrations. [PubMed: 17717283]

49--. Nobili V, Alkhouri N, Bartuli A, et al. Severity of liver injury and atherogenic lipid profile in children with nonalcoholic fatty liver disease. Pediatr Res. 2010; 67:665-670. First study to demonstrate that the histological severity of nonalcoholic fatty liver disease (NAFLD) in children correlates with higher lipid ratios and a more atherogenic lipid panel. [PubMed: 20496475]

50. Alkhouri N, Tamimi TA, Yerian L, Lopez R, Zein NN, Feldstein AE. The inflamed liver and atherosclerosis: a link between histologic severity of nonalcoholic fatty liver disease and increased cardiovascular risk. Dig Dis Sci. 2009; 55(9):2644-2650. [PubMed: 19960252]

51. Kantartzis K, Rittig K, Cegan A, et al. Fatty liver is independently associated with alterations in circulating HDL2 and HDL3 subfractions. Diabetes Care. 2008; 31:366-368. [PubMed: 18000185]

52. Garcia-Sanchez C, Torres-Tamayo M, Juarez-Meavepena M, et al. Lipid plasma concentrations of HDL subclasses determined by enzymatic staining on polyacrylamide electrophoresis gels in children with metabolic syndrome. Clin Chim Acta. 2011; 30:292-298. [PubMed: 21036160]

53. Perez-Mendez O, Torres-Tamayo M, Posadas-Romero C, et al. Abnormal HDL subclasses distribution in overweight children with insulin resistance or type 2 diabetes mellitus. Clin Chim Acta. 2007; 376:17-22. [PubMed: 16934792]

54. Chalasani N, Deeg MA, Crabb DW. Systemic levels of lipid peroxidation and its metabolic and dietary correlates in patients with nonalcoholic steatohepatitis. Am J Gastroenterol. 2004; 99:1497-1502. [PubMed: 15307867]

55. Musso G, Gambino R, Biroli G, et al. Hypoadiponectinemia predicts the severity of hepatic fibrosis and pancreatic $\beta$-cell dysfunction in nondiabetic nonobese patients with nonalcoholic steatohepatitis. Am J Gastroenterol. 2005; 100:2438-2446. [PubMed: 16279898]

56. Pagano C, Soardo G, Esposito W, et al. Plasma adiponectin is decreased in nonalcoholic fatty liver disease. Eur J Endocrinol. 2005; 152:113-118. [PubMed: 15762194]

57. Targher G. Relationship between high-sensitivity C-reactive protein levels and liver histology in subjects with non-alcoholic fatty liver disease. J Hepatol. 2006; 45:879-881. author reply 881 872. [PubMed: 17049665]

58. Targher G, Bertolini L, Scala L, et al. Plasma PAI-1 levels are increased in patients with nonalcoholic steatohepatitis. Diabetes Care. 2007; 30:E31-E32. [PubMed: 17468361]

59. Pepys MB, Hirschfield GM. C-reactive protein: a critical update. J Clin Invest. 2003; 111:18051812. [PubMed: 12813013]

60. Yoneda M, Mawatari H, Fujita K, et al. High-sensitivity C-reactive protein is an independent clinical feature of nonalcoholic steatohepatitis (NASH) and also of the severity of fibrosis in NASH. J Gastroenterol. 2007; 42:573-582. [PubMed: 17653654]

61. Pacifico L, Anania C, Martino F, et al. Functional and morphological vascular changes in pediatric nonalcoholic fatty liver disease. Hepatology. 2010; 52:1643-1651. [PubMed: 20890890] 
62. Manco M, Marcellini M, Giannone G, Nobili V. Correlation of serum TNF- $\alpha$ levels and histologic liver injury scores in pediatric nonalcoholic fatty liver disease. Am J Clin Pathol. 2007; 127:954960. [PubMed: 17509993]

63. Kawano J, Arora R. The role of adiponectin in obesity, diabetes, and cardiovascular disease. J Cardiometab Syndr. 2009; 4:44-49. [PubMed: 19245516]

64. Tian L, Luo N, Klein RL, Chung BH, Garvey WT, Fu Y. Adiponectin reduces lipid accumulation in macrophage foam cells. Atherosclerosis. 2009; 202:152-161. [PubMed: 18511057]

65. Liu YL, Liang HR, Liu HT, et al. Association of serum adiponectin levels with artherosclerosis and the metabolic syndrome in obese children. J Pediatr Endocrinol Metab. 2010; 23:743-751. [PubMed: 21073115]

66. Lebensztejn DM, Wojtkowska M, Skiba E, Werpachowska I, Tobolczyk J, Kaczmarski M. Serum concentration of adiponectin, leptin and resistin in obese children with non-alcoholic fatty liver disease. Adv Med Sci. 2009; 54:177-182. [PubMed: 20022856]

67. Bugianesi E, Pagotto U, Manini R, et al. Plasma adiponectin in nonalcoholic fatty liver is related to hepatic insulin resistance and hepatic fat content, not to liver disease severity. J Clin Endocrinol Metab. 2005; 90:3498-3504. [PubMed: 15797948]

68. Duffy BK, Gurm HS, Rajagopal V, Gupta R, Ellis SG, Bhatt DL. Usefulness of an elevated neutrophil to lymphocyte ratio in predicting long-term mortality after percutaneous coronary intervention. Am J Cardiol. 2006; 97:993-996. [PubMed: 16563903]

69. Azab B, Zaher M, Weiserbs KF, et al. Usefulness of neutrophil to lymphocyte ratio in predicting short- and long-term mortality after non-ST-elevation myocardial infarction. Am J Cardiol. 2010; 106:470-476. [PubMed: 20691303]

70. Gibson PH, Cuthbertson BH, Croal BL, et al. Usefulness of neutrophil/lymphocyte ratio as predictor of new-onset atrial fibrillation after coronary artery bypass grafting. Am J Cardiol. 2010; 105:186-191. [PubMed: 20102916]

71. Tamhane UU, Aneja S, Montgomery D, Rogers EK, Eagle KA, Gurm HS. Association between admission neutrophil to lymphocyte ratio and outcomes in patients with acute coronary syndrome. Am J Cardiol. 2008; 102:653-657. [PubMed: 18773982]

72. Nascimento H, Rocha S, Rego C, et al. Leukocyte count versus C-reactive protein levels in obese portuguese patients aged 6-12 years old. Open Biochem J. 2010; 4:72-76. [PubMed: 20676221]

73. Alkhouri N, Morris-Stiff G, Campbell C, et al. Usefulness of neutrophil to lymphocyte ratio in predicting steatohepatitis in patients with nonalcoholic fatty liver disease. Am J Gastroenterol. 2010; 105:S110.

74. Chu SG, Becker RC, Berger PB, et al. Mean platelet volume as a predictor of cardiovascular risk: a systematic review and meta-analysis. J Thromb Haemost. 2010; 8:148-156. [PubMed: 19691485]

75. Tavil Y, Sen N, Yazici H, et al. Coronary heart disease is associated with mean platelet volume in Type 2 diabetic patients. Platelets. 2010; 21:368-372. [PubMed: 20521863]

76. Arslan N, Makay B. Mean platelet volume in obese adolescents with nonalcoholic fatty liver disease. J Pediatr Endocrinol Metab. 2010; 23:807-813. [PubMed: 21073123]

77. Brea A, Mosquera D, Martin E, Arizti A, Cordero JL, Ros E. Nonalcoholic fatty liver disease is associated with carotid atherosclerosis: a case-control study. Arterioscler Thromb Vasc Biol. 2005; 25:1045-1050. [PubMed: 15731489]

78. Targher G, Bertolini L, Rodella S, et al. Nonalcoholic fatty liver disease is independently associated with an increased incidence of cardiovascular events in Type 2 diabetic patients. Diabetes Care. 2007; 30:2119-2121. [PubMed: 17519430]

79. Sookoian S, Pirola CJ. Non-alcoholic fatty liver disease is strongly associated with carotid atherosclerosis: a systematic review. J Hepatol. 2008; 49:600-607. [PubMed: 18672311]

80. Targher G, Bertolini L, Padovani R, et al. Relations between carotid artery wall thickness and liver histology in subjects with nonalcoholic fatty liver disease. Diabetes Care. 2006; 29:1325-1330. [PubMed: 16732016]

81. Villanova N, Moscatiello S, Ramilli S, et al. Endothelial dysfunction and cardiovascular risk profile in nonalcoholic fatty liver disease. Hepatology. 2005; 42:473-480. [PubMed: 15981216]

82-. Pacifico L, Cantisani V, Ricci P, et al. Nonalcoholic fatty liver disease and carotid atherosclerosis in children. Pediatr Res. 2008; 63:423-427. Demonstrates that the severity of fatty liver as 
assessed by ultrasound is significantly associated with maximum carotid intima-media thickness. [PubMed: 18356751]

83. Demircioglu F, Kocyigit A, Arslan N, Cakmakci H, Hizli S, Sedat AT. Intima-media thickness of carotid artery and susceptibility to atherosclerosis in obese children with nonalcoholic fatty liver disease. J Pediatr Gastroenterol Nutr. 2008; 47:68-75. [PubMed: 18607271]

84. Kelishadi R, Cook SR, Amra B, Adibi A. Factors associated with insulin resistance and nonalcoholic fatty liver disease among youths. Atherosclerosis. 2009; 204:538-543. [PubMed: 19013572]

85. Schwimmer JB, Deutsch R, Behling C, Lavine JE. Fatty liver as a determinent of atherosclerosis. Hepatology. 2005; 42:610A.

86. Imazeki T, Sato Y, Inoue F, et al. Evaluation of coronary artery remodeling in patients with acute coronary syndrome and stable angina by multislice computed tomography. Circ J. 2004; 68:10451050. [PubMed: 15502387]

87. Akabame S, Hamaguchi M, Tomiyasu K, et al. Evaluation of vulnerable coronary plaques and nonalcoholic fatty liver disease (NAFLD) by 64-detector multislice computed tomography (MSCT). Circ J. 2008; 72:618-625. [PubMed: 18362435]

88. Gomez Rosso L, Benitez MB, Fornari MC, et al. Alterations in cell adhesion molecules and other biomarkers of cardiovascular disease in patients with metabolic syndrome. Atherosclerosis. 2008; 199:415-423. [PubMed: 18096167]

89. Sookoian S, Castano GO, Burgueno AL, et al. Circulating levels and hepatic expression of molecular mediators of atherosclerosis in nonalcoholic fatty liver disease. Atherosclerosis. 2010; 209:585-591. [PubMed: 19896127]

90. Lucero D, Zago V, Lopez GI, et al. Pro-inflammatory and atherogenic circulating factors in nonalcoholic fatty liver disease associated to metabolic syndrome. Clin Chim Acta. 2011; 412:143147. [PubMed: 20887718]

91. Ito S, Yukawa T, Uetake S, Yamauchi M. Serum intercellular adhesion molecule-1 in patients with nonalcoholic steatohepatitis: comparison with alcoholic hepatitis. Alcohol Clin Exp Res. 2007; 31:S83-S87. [PubMed: 17331172]

92. Adams LA, Lymp JF, St Sauver J, et al. The natural history of nonalcoholic fatty liver disease: a population-based cohort study. Gastroenterology. 2005; 129:113-121. [PubMed: 16012941]

93. Matteoni CA, Younossi ZM, Gramlich T, Boparai N, Liu YC, McCullough AJ. Nonalcoholic fatty liver disease: a spectrum of clinical and pathological severity. Gastroenterology. 1999; 116:14131419. [PubMed: 10348825]

94. Sanyal AJ, Banas C, Sargeant C, et al. Similarities and differences in outcomes of cirrhosis due to nonalcoholic steatohepatitis and hepatitis C. Hepatology. 2006; 43:682-689. [PubMed: 16502396]

95. Ioannou GN, Weiss NS, Boyko EJ, Mozaffarian D, Lee SP. Elevated serum alanine aminotransferase activity and calculated risk of coronary heart disease in the United States. Hepatology. 2006; 43:1145-1151. [PubMed: 16628637]

96. Lee DS, Evans JC, Robins SJ, et al. $\gamma$-glutamyl transferase and metabolic syndrome, cardiovascular disease, and mortality risk: the Framingham Heart Study. Arterioscler Thromb Vasc Biol. 2007; 27:127-133. [PubMed: 17095717]

97. Schindhelm RK, Dekker JM, Nijpels G, et al. Alanine aminotransferase predicts coronary heart disease events: a 10-year follow-up of the Hoorn Study. Atherosclerosis. 2007; 191:391-396. [PubMed: 16682043]

98. van Vliet M, von Rosenstiel IA, Schindhelm RK, Brandjes DP, Beijnen JH, Diamant M. The association of elevated alanine aminotransferase and the metabolic syndrome in an overweight and obese pediatric population of multi-ethnic origin. Eur J Pediatr. 2009; 168:585-591. [PubMed: 18696112]

99. Wei C, Ford A, Hunt L, Crowne EC, Shield JP. Abnormal liver function in children with metabolic syndrome from a UK-based obesity clinic. Arch Dis Child. 2010 Epub ahead of print.

100. St George A, Bauman A, Johnston A, Farrell G, Chey T, George J. Effect of a lifestyle intervention in patients with abnormal liver enzymes and metabolic risk factors. J Gastroenterol Hepatol. 2009; 24:399-407. [PubMed: 19067776] 
101. Nobili V, Manco M, Devito R, et al. Lifestyle intervention and antioxidant therapy in children with nonalcoholic fatty liver disease: a randomized, controlled trial. Hepatology. 2008; 48:119_ 128. [PubMed: 18537181]

102-.. Lavine JE, Schwimmer JB, Molleston JP, et al. Vitamin E, metformin or placebo for treatment of nonalcoholic fatty liver disease in children. Hepatology. 2010; 52:374A. Largest randomized control study assessing the effectiveness of vitamin $\mathrm{E}$ or metformin in children with biopsyproven NAFLD. 


\section{Executive summary}

\section{Nonalcoholic fatty liver disease in children}

- Nonalcoholic fatty liver disease (NAFLD) is the most common form of chronic liver disease in children and adolescents.

- It is strongly associated with the metabolic syndrome and insulin resistance, which are established risk factors for cardiovascular (CV) disease.

\section{NAFLD \& CV risk}

- NAFLD confers an increased CV risk independent of the metabolic syndrome and its individual components.

- Patients with the advanced form of NAFLD, nonalcoholic steatohepatitis, may have higher $\mathrm{CV}$ risk than patients with simple steatosis.

\section{Atherogenic dyslipidemia \& NAFLD}

- Liver steatosis is associated with a proatherogenic lipid profile characterized by small dense LDL and larger VLDL.

- Children with NAFLD have higher total cholesterol, LDL and triglycerides, and lower HDL levels.

- The histologic severity of liver injury in children with NAFLD correlates positively with higher traditional lipid ratios and a more atherogenic lipid panel.

\section{Other markers of $\mathrm{CV}$ risk in children with NAFLD}

- Markers of systemic inflammation such as C-reactive protein, TNF and neutrophil to lymphocyte ratio are increased in nonalcoholic steatohepatitis.

- Endothelial dysfunction is evident in patients with NAFLD as measured by intima-media thickness and circulating levels of adhesion molecules.

- Epidemiological studies have shown an increased prevalence of CV disease in NAFLD patients.

\section{Therapeutic interventions}

- Lifestyle interventions with diet and physical activity may lead to improvement in NAFLD and dyslipidemia in children with NAFLD

- Vitamin E, metformin, omega-3 fatty acids and statins may represent efficient novel therapeutic approaches in the treatment of children with NAFLD 


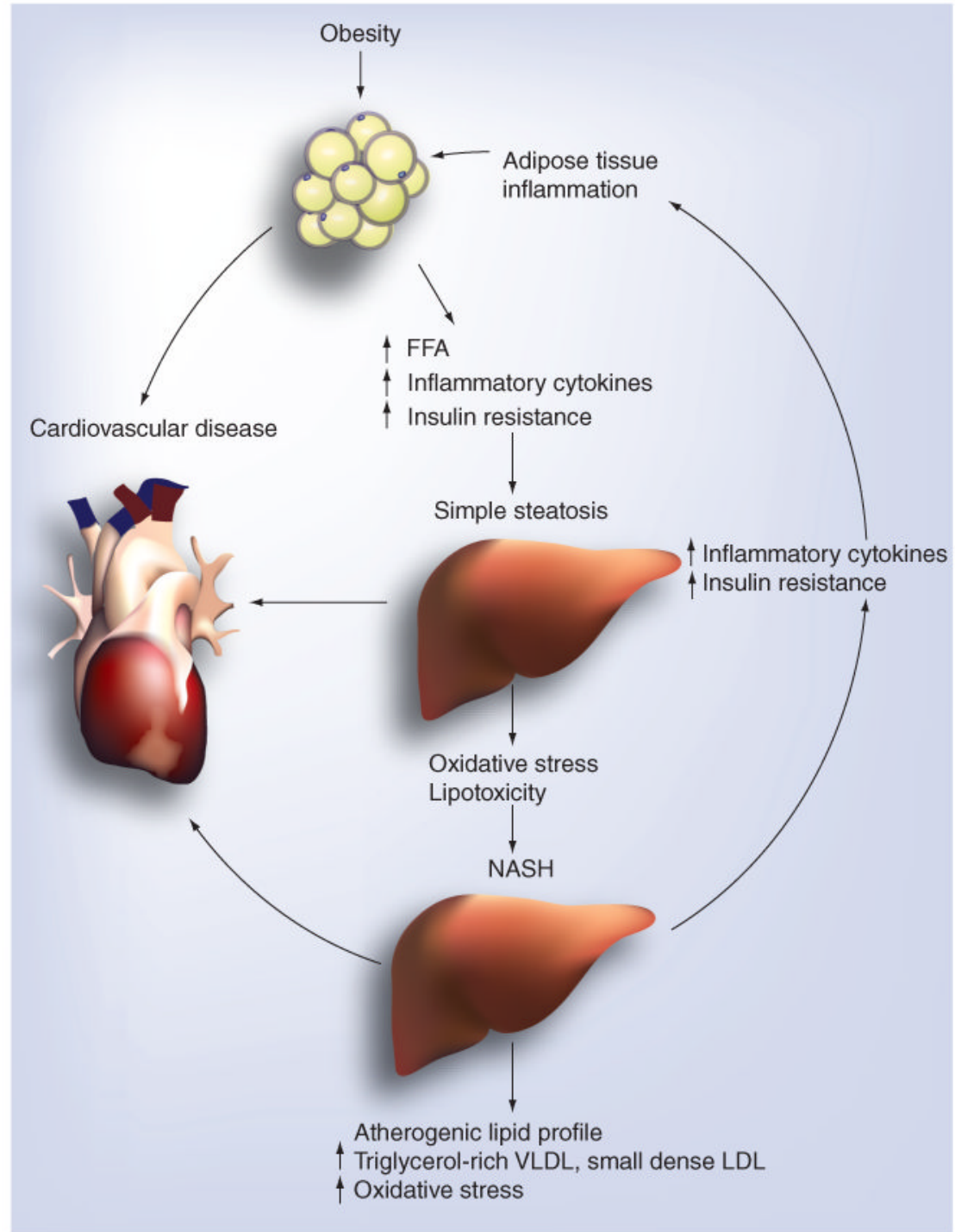

Figure 1.

Mechanisms linking obesity, fatty liver and atherosclerosis. Obesity is associated with expansion of adipose tissue and a state of chronic inflammation. This leads to insulin resistance and the release of FFA into the circulation, which results in the development of fatty liver. Further inflammation and oxidative stress are responsible for the progression of fatty liver to NASH, which leads to further insulin resistance, systemic inflammation and an atherogenic lipid profile; all risk factors for cardiovascular disease.

FFA: Free fatty acid; NASH: Nonalcoholic steatohepatitis. 